\title{
The cost of a combination Anti-Retroviral Therapy (cART) optimization pathway as maintenance therapy in HIV-1 infected patients
}

Roberto Ravasio ${ }^{1}$, Fabio Rigo ${ }^{2}$, Emanuela Lattuada ${ }^{2}$, Ercole Concia ${ }^{2}$, Massimiliano Lanzafame ${ }^{2}$

${ }^{1}$ Health Publishing \& Services, Milano - Italy

${ }^{2}$ Infection Disease Unit, G.B Rossi Hospital, Verona - Italy

\begin{abstract}
BACKGROUND: In order to reduce/prevent combination Anti-Retroviral Therapy (cART)-related toxicity, while maintaining its therapeutic effectiveness over time, the optimization of the antiretroviral therapy could be performed.

AIM: To estimate the economic impact on the Italian National Health Service (NHS) of a cART optimization pathway as maintenance therapy in HIV-1 infected patients over one-year period.

METHODS: Patient data were retrieved from the electronic medical record system in use (year 2015) in a reference HIV Center in Northern Italy. The analysis considered naïve patients and non-naïve patients. To estimate the actual ART expenditure charged to the Center we calculated the cost of cART received during 12 months for each patient. Subsequently, referring to the same patients, a "potential" cART expenditure was estimated. This potential expenditure was estimated taking in consideration the adoption of a specific optimization pathway aimed at maintaining over the time the cART efficacy. Lastly, to assess the sustainability of the optimization pathway, we compared the actual cART expenditure with the potential one. We considered only drug costs (ex-factory prices, included all discounts and VAT) from the perspective of the Italian NHS.

RESULTS: In the 2015, the total expenditure for 564 enrolled HIV-1 patients treated with cART was $€ 4,042,983$. The mean treatment cost per patient was $€ 7,168$. If the Center adopted a specific optimization pathway, the total expenditure would be $€ 3,914,855$ (-€ 128,128).

CONCLUSIONS: From the Italian NHS's perspective, the adoption of a specific cART optimization pathway represents a cost-saving option as maintenance antiretroviral therapy in HIV-1 infected patients.
\end{abstract}

Keywords

Combination Anti-Retroviral Therapy - cART; HIV-1; Italian National Health System

\section{INTRODUCTION}

The Continuum of Care in the management of HIV infection (Human Immunodeficiency Virus) describes a patient care pathway that begins with the diagnosis, and then continues with the choice of therapy (engagement in care) and its maintenance (retention in care). The selection of the antiretroviral therapy is the foundation of this pathway. The latest Italian Guidelines recommend the combination of two NRTIs (Nucleoside Reverse Transcriptase Inhibitor) with a INI (INtegrase Inhibitor) or a NNRTI (Non-Nucleoside Reverse Transcriptase Inhibitor), or alterna- tively in particular conditions a PI (Protease Inhibitor), boosted with ritonavir or cobicistat, as a standard treatment for HIV-infected patients [1]. These combination therapies are defined by the term cART (combination AntiRetroviral Therapy) [1].

Despite the great progress achieved by the pharmacological research, a therapy capable of eradicating HIV remains a mirage. It is therefore crucial to maintain the effectiveness over time of the antiretroviral treatment adopted, despite the fact that the administration of a cART regimen could, in the medium to long term, result in the reduction in adherence, the onset of toxicity, the aggravation of
Corresponding author Roberto Ravasio rravasio@aboutpharma.com 
already present comorbidities, an increase in the risk of pharmacological interactions with concomitant therapies due to contingent comorbidities, or the loss of strength/evidence (recommendation) of the regimen administered [1-3].

In order to reduce/prevent cART-related toxicity, while maintaining its therapeutic effectiveness over time, the optimization of the antiretroviral therapy could be performed, as indicated in the Italian Guidelines [1]. Three optimization options can be assumed. The first involves the reduction in the number of antiretroviral regimens administered; in this case there would be a switch from a threedrug (triple) to a two-drug (double) therapy. The second option is not aimed at changing the current therapy, but to reduce the number of daily doses/administrations and/or daily tablets. The third option, finally, involves the replacement of the current regimen with other regimens consisting of the same number of drugs or more. For each of the three options, the modification of the cART therapy should occur under virological suppression (HIVRNA $<50$ copies/ml) [1].

Without addressing here the issue of the clinical implications - since they are widely discussed in the Italian Guidelines, to which it is hereby referred to [1] - the optimization of the cART regimens presents a further issue, concerning economic sustainability. If, as seen from past experience [4], the switch from a three-drug (triple) to a two-drug (double) therapy can result in a reduction in the expenditure on antiretroviral drugs, the same cannot be said for the other optimization pathways presented. For example, with regard to the potential cardiovascular and/or metabolic toxicity associated with current antiretroviral regimens, the Italian Guidelines suggest instead to preferably adopt regimens based on integrase inhibitors (and not on protease inhibitors), without however saying anything about their economic sustainability [1].

The objective of this analysis is therefore to ascertain whether and how a cART optimization pathway can, as a whole, be sustainable for the Italian National Health Service.

\section{METHODS}

\section{Analysis design}

An observational, retrospective and non-interventional analysis was conducted. The statistical source consists of all HIV-diagnosed patients (hereinafter referred to as "patients treated", or even more concisely as "patients") of the Infectious Diseases Operating Unit of the Hospital "G.B. Rossi" in Verona (hereinafter referred to as the "Center"), who received an antiretroviral therapy during the period $1^{\text {st }}$ January- $31^{\text {st }}$ December 2015.

The patients (who in the analysis were identified by encrypted - and therefore strictly anonymous - codes) were classified into two groups: i) naïve, i.e. patients who started the antiretroviral treatment during the observation year, and ii) non-naïve, i.e. patients who were already being treated with an antiretroviral regimen.

For each of them - characterized by the main demographic (mean age, gender, etc.) and clinical variables (virological suppression, resistances, $\mathrm{HCV}, \mathrm{HBV}$, etc.) - the antiretroviral therapy received during the last 12 months was identified, in order to calculate its actual pharmaceutical cost borne by the Center. Subsequently, with reference to the same patients, an antiretroviral expenditure was estimated; this was defined as "potential", because it was due to the adoption of a specific optimization pathway aimed at improving the maintenance of the antiretroviral therapy effectiveness over time [1]. The comparison between actual and potential spending finally allowed to express a judgment on the sustainability (or not) of the optimization pathway with regard to the cART regimens administered during the year 2015.

\section{Optimization pathway}

The optimization pathway suggested here was constructed in accordance with the indications of the Italian Guidelines [1]. Two optimization options were assumed, in chronological order. The first is the switch from a three-drug to a two-drug regimen. Based on the results of a previous experience gained by the Center, the two-drug regimen selected was the combination nevirapine $400 \mathrm{mg}$ /day (NNRTI) and raltegravir $400 \mathrm{mg}$ bis in die, BID (INI) [4]. The therapy switch could only be executed for patients who had been in virological suppression (viral load $<50$ copies/ $\mathrm{ml}$ ) for at least 12 months, without any prior exposure to an INI, with no NNRTI-associated resistance mutations and with a toxicity (dyslipidemia and/or chronic renal failure) correlated with the therapy received.

Since - in view of the potential cardiovascular and/or metabolic toxicity of the current cART regimens - the Italian Guidelines suggest instead to preferably adopt regimens based on INIs, the second optimization pathway option involves, for the subjects who do not fall under the first option, the substitution of the toxic regimens with others, less toxic [1]. The identification of the potentially toxic regimens was performed by analyzing the cART therapy received by each patient. Then, based on the history of cART received 
and the indications suggested by the Italian Guidelines, a number of possible alternative regimens were assumed [1]. In this case too, the therapy switch had to take place in conditions of virological suppression maintained for at least one year. Naïve patients were not included in the optimization pathway because they have not been in virological suppression for at least 12 months since they received the first antiretroviral treatment during the observation year (2015).

\section{Treatment cost}

The two expenditures - actual and potential - for the antiretroviral drugs were calculated considering the purchase prices, net of all (mandatory and not) discounts and including VAT, borne by the Hospital Pharmacy of the Center. All costs are referred to the year 2015.
In case of switch from a three-drug regimen to that with nevirapine/raltegravir, for the latter a mean annual treatment cost of $€ 5,566$ was considered [4]. In case of replacement of the potentially toxic antiretroviral regimen (cardiovascular and/or metabolic effect), an average annual cost calculated on the basis of the antiretroviral regimens suggested as alternatives was estimated for each patient [1].

\section{Data analysis}

Quantitative variables have been described as mean value ( \pm standard deviation), categorical variables as numeric value (percentage). The significance of the differences between the data found/processed was verified by applying the (two-tier) Student's t-test. The analysis was supported by Microsoft ${ }^{\circledR}$ Excel $^{\circledR}$ for Windows ${ }^{\circledR}$ (Microsoft Corporation,

\begin{tabular}{|c|c|c|c|}
\hline Characteristics & Naïve & Non naïve & Total \\
\hline Patients, $\mathrm{n}$. & 34 & 530 & 564 \\
\hline Mean age, years ( \pm SD) & $41.9( \pm 9.8)$ & $49.0( \pm 9.7)$ & $48.5( \pm 9.9)$ \\
\hline Time to diagnosis, years $( \pm \mathrm{SD})$ & $5.2( \pm 5.1)$ & $13.7( \pm 8.1)$ & $13.2( \pm 8.2)$ \\
\hline Previous treatments failed, $n .( \pm S D)$ & $0.0( \pm 0.0)$ & $0.6( \pm 1.2)$ & $0.6( \pm 1.2)$ \\
\hline \multicolumn{4}{|l|}{ Virological suppression'1 n. (\%) } \\
\hline - For more than 1 year & $0(0.0)$ & $405(76.4)$ & $405(71.8)$ \\
\hline - For less than 1 year & $1(2.9)$ & $16(3.0)$ & $17(3.0)$ \\
\hline • No & $33(97.1)$ & $109(20.6)$ & $142(25.2)$ \\
\hline CD4, n. ( $\pm \mathrm{SD})$ & $394.1( \pm 179.0)$ & $625.2( \pm 283.3)$ & $611.2( \pm 283.4)$ \\
\hline \%CD4, n. ( \pm SD) & $20.3 \%( \pm 9.4 \%)$ & $28.9 \%( \pm 9.5 \%)$ & $28.4 \%( \pm 9.7 \%)$ \\
\hline \multicolumn{4}{|l|}{ Resistances, n. (\%) } \\
\hline • No & $24(70.6)$ & $168(31.7)$ & $192(34.0)$ \\
\hline - Resistance/partial resistance & $4(11.8)$ & $127(24.0)$ & $131(23.2)$ \\
\hline - Test not performed/unavailable & $6(17.6)$ & $235(44.3)$ & $241(42.7)$ \\
\hline \multicolumn{4}{|l|}{ HLA B5701, n. (\%) } \\
\hline - Positive & $1(2.9)$ & $8(1.5)$ & $9(1.6)$ \\
\hline - Negative & $20(58.8)$ & $344(64.9)$ & $364(64.5)$ \\
\hline - Test not performed/unavailable & $13(38.2)$ & $178(33.6)$ & $191(33.9)$ \\
\hline Smoker, n. (\%) & $13(38.2)$ & $151(28.5)$ & $164(29.1)$ \\
\hline Systolic pressure, mmHg ( \pm SD) & $126.0( \pm 10.1)$ & $128.9( \pm 11.5)$ & $128.8( \pm 11.5)$ \\
\hline Diastolic pressure, $\mathrm{mmHg}( \pm \mathrm{SD})$ & $80.7( \pm 5.1)$ & $82.5( \pm 7.4)$ & $82.4( \pm 7.3)$ \\
\hline HCV, n. (\%) & $3(8.8)$ & $134(25.3)$ & $137(24.3)$ \\
\hline HBV, n. (\%) & $0(0.0)$ & $18(3.4)$ & $18(3.2)$ \\
\hline Dyslipidemia, n. (\%) & $6(17.6)$ & $189(35.7)$ & $195(34.6)$ \\
\hline Total cholesterol, mg/dl ( \pm SD) & $176.0( \pm 42.3)$ & $188.8( \pm 41.4)$ & $188.0( \pm 41.5)$ \\
\hline HDL cholesterol, mg/dl ( \pm SD) & $47.0( \pm 14.6)$ & $51.8( \pm 17.4)$ & $51.5( \pm 17.3)$ \\
\hline Chronic renal failure, n. (\%) & $1(2.9)$ & $20(3.8)$ & $21(3.7)$ \\
\hline \multicolumn{4}{|l|}{ Densitometry, n. (\%) } \\
\hline - No & $34(100.0)$ & $465(87.7)$ & $499(88.5)$ \\
\hline - Osteopenia & $0(0.0)$ & $50(9.4)$ & $50(8.9)$ \\
\hline - Osteoporosis & $0(0.0)$ & $15(2.8)$ & $15(2.7)$ \\
\hline Previous CVD event, n. (\%) & $0(0.0)$ & $23(4.3)$ & $23(4.1)$ \\
\hline Dyslipidemia or osteoporosis drugs, n. (\%) & $1(2.9)$ & $192(36.2)$ & $193(34.2)$ \\
\hline Hypertension drugs, n. (\%) & $1(2.9)$ & $96(18.1)$ & $97(17.2)$ \\
\hline
\end{tabular}

Table I. Main demographic and clinical characteristics of the patients enrolled ${ }^{1}$ HIV-RNA $<50$ copies/ml 
Seattle, WA, USA) and SPSS ${ }^{\circledR} 13.0$ for Windows $^{\circledR}$ (SPSS Inc., Chicago, IL, USA).

\section{RESULTS}

\section{Characteristics of the sample}

Overall, during the year 2015, 564 HIV-diagnosed patients under treatment with cART regimens were referred to the Center. Of these, $6 \%(\mathrm{n} .=34)$ started antiretroviral therapy in 2015 (naïve patients). Table I shows the main demographic and clinical characteristics of the patients enrolled. The mean age was $48.5( \pm 9.9)$ years, with an average duration of HIV infection at diagnosis of $13.2( \pm$ $8.2)$ years. $71.8 \%(n .=405)$ of patients had been in virological suppression for at least 12 months.

\section{CART regimens}

$76.4 \%$ (n. $=431)$ of patients received a regimen consisting of at least three antiretrovi-

\begin{tabular}{|c|c|c|c|c|c|c|c|c|c|}
\hline \multirow{3}{*}{ ART regimens } & \multicolumn{9}{|c|}{ Patients } \\
\hline & \multicolumn{3}{|c|}{ Naïve } & \multicolumn{3}{|c|}{ Non-naïve } & \multicolumn{3}{|c|}{ Total } \\
\hline & n. & $\%$ & Mean cost $(€)$ & n. & $\%$ & Mean cost $(€)$ & n. & $\%$ & Mean cost $(€)$ \\
\hline $2 \mathrm{NRTI}+1 \mathrm{PI}^{1}$ & 5 & 14.7 & 4,811 & 181 & 34.2 & 9,195 & 186 & 33.0 & 9,077 \\
\hline $2 \mathrm{NRTI}+1 \mathrm{NNRTI}^{1}$ & 16 & 47.1 & 3,092 & 149 & 28.1 & 6,397 & 165 & 29.3 & 6,076 \\
\hline $3 \mathrm{NRTI}^{1}$ & 2 & 5.9 & 1,518 & 42 & 7.9 & 6,478 & 44 & 7.8 & 6,252 \\
\hline $2 \mathrm{NRTI}+1 \mathrm{INI}^{1}$ & - & - & - & 35 & 6.6 & 10,119 & 35 & 6.2 & 10,119 \\
\hline $1 \mathrm{NRTI}+1 \mathrm{PI}$ & 1 & 2.9 & 2,107 & 34 & 6.4 & 5,773 & 35 & 6.2 & 5,668 \\
\hline $1 \mathrm{PI}$ & - & - & - & 27 & 5.1 & 4,455 & 27 & 4.8 & 4,455 \\
\hline $1 \mathrm{NNRTI}+1 \mathrm{INI}$ & - & - & - & 13 & 2.5 & 4,657 & 13 & 2.3 & 4,657 \\
\hline $1 \mathrm{INI}+1 \mathrm{PI}$ & - & - & - & 13 & 2.5 & 10,571 & 13 & 2.3 & 10,571 \\
\hline $1 \mathrm{NRTI}+1 \mathrm{INI}$ & - & - & - & 11 & 2.1 & 6,388 & 13 & 2.3 & 3,960 \\
\hline $2 \mathrm{NRTI}$ & - & - & - & 10 & 1.9 & 3,768 & 11 & 2.0 & 6,388 \\
\hline $1 \mathrm{NRTI}+1 \mathrm{NNRTI}$ & - & - & - & 9 & 1.7 & 2,146 & 10 & 1.8 & 3,768 \\
\hline $1 \mathrm{INI}$ & 10 & 29.4 & 2,960 & 3 & 0.6 & 7,295 & 9 & 1.6 & 2,146 \\
\hline $1 \mathrm{NRTI}+1 \mathrm{INI}+1 \mathrm{PI}$ & - & - & - & 1 & 0.2 & 11,911 & 1 & 0.2 & 11,911 \\
\hline $1 \mathrm{NNRTI}$ & - & - & - & 1 & 0.2 & 432 & 1 & 0.2 & 432 \\
\hline 1 CCR5 + 1 PI & - & - & - & 1 & 0.2 & 15,134 & 1 & 0.2 & 15,134 \\
\hline Total & 34 & 100 & 3,184 & 530 & 100 & 7,424 & 564 & 100 & 7,424 \\
\hline
\end{tabular}

Table II. CART regimens administered

${ }^{1}$ Highly Active Anti-Retroviral Therapy (HAART) regimens

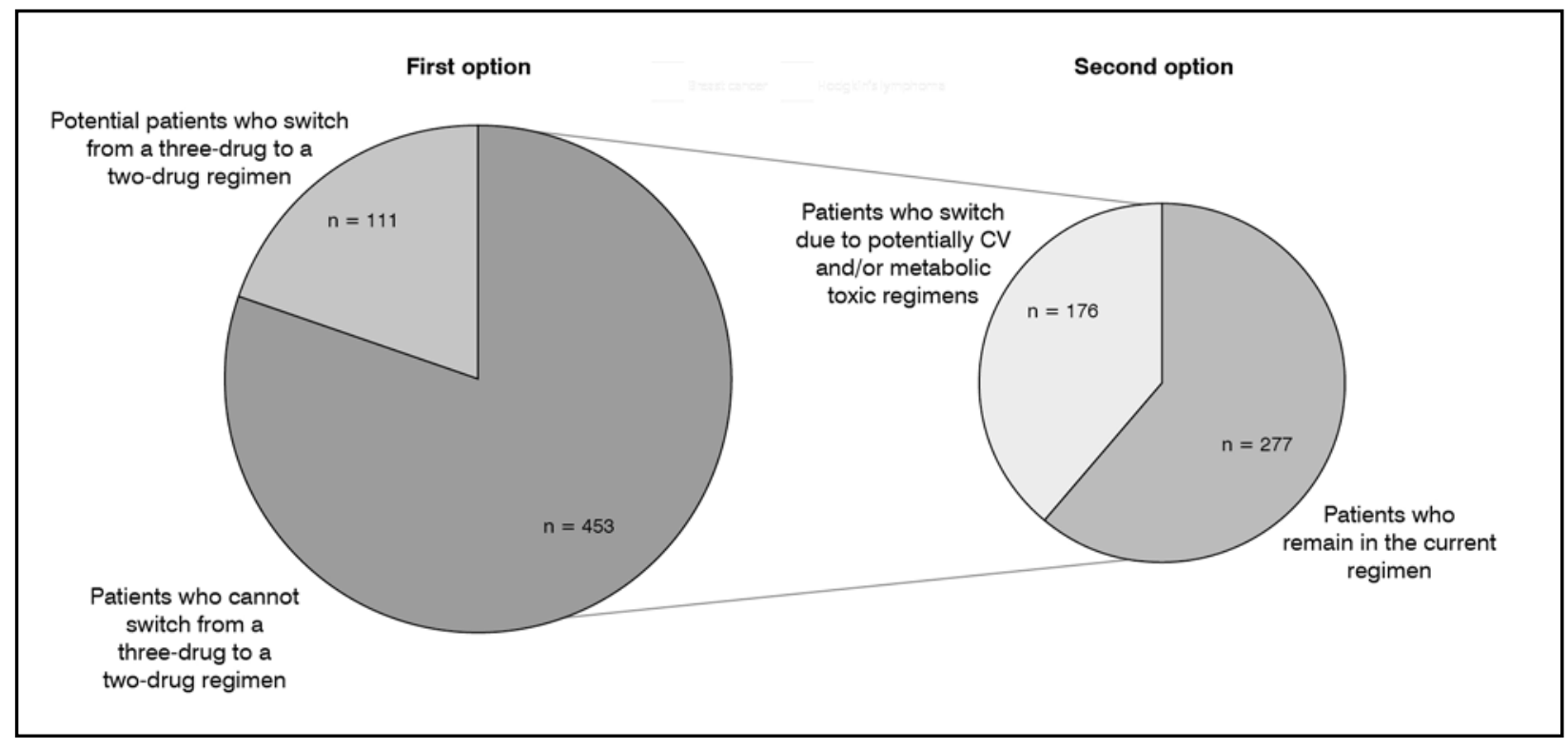

Figure 1. Optimization pathway 
ral drugs (naïve: $67.6 \%$, non-naïve: $77.0 \%$ ) (Table II). The most frequently used regimens were 2 NRTIs +1 PI (n. $=186 ; 33.0 \%$; $€ 9,077)$ and 2 NRTIs +1 NNRTI (n. $=165$; $29.3 \%$; $€ 6,076$ ). $46.6 \%$ of the regimens administered contained a PI, while $15.2 \%$ contained an INI. The average duration of treatment during the year for naïve patients was 5.4 ( \pm 3.2) months, whereas for non-naïve patients it was 12 months (all non-naïve patients were in continuous treatment for the 12 months of observation).

\section{Optimization pathway}

Following the chronological sequence of the potential therapy switches assumed within the cART optimization pathway, in 111 patients it would have been possible to switch from a three-drug regimen to ne- virapine/raltegravir (Figure 1). Of these, 53 were in treatment with 2 NRTIs +1 PI, 51 with 2 NRTIs +1 NNRTI and 7 received 3 NRTIs. All 111 patients had been in virological suppression for at least 12 months, without any NNRTI- or INI-associated resistance mutations. Each of them also presented a toxicity due to dyslipidemia $\left(95.5 \%, n_{.}=106\right)$ and/or chronic renal failure $(7.2 \%, \mathrm{n} .=8)$.

Of the remaining 453 patients, 176 were the subjects for whom it would have been desirable to replace the adopted regimen (Table III), characterized by a potential cardiovascular and/or metabolic toxicity, with one of those indicated in the Italian Guidelines (Table IV). On average, about 4 alternative regimens for each patient were provided for.

\begin{tabular}{|c|c|c|c|c|}
\hline \multirow{2}{*}{ Agents } & \multirow{2}{*}{ ART Regimens } & \multicolumn{3}{|c|}{ Patients } \\
\hline & & n. & $\%$ & Mean cost $(€)$ \\
\hline Atazanavir Maraviroc Ritonavir & $1 \mathrm{CCR} 5+1 \mathrm{PI}$ & 1 & 0.6 & 15,134 \\
\hline Raltegravir Lopinavir/Ritonavir & $1 \mathrm{INI}+1 \mathrm{PI}$ & 1 & 0.6 & 3,706 \\
\hline Raltegravir Darunavir Ritonavir & $1 \mathrm{INI}+1 \mathrm{PI}$ & 7 & 4.0 & 11,224 \\
\hline Darunavir Ritonavir & $1 \mathrm{INI}+1 \mathrm{PI}$ & 2 & 1.1 & 12,203 \\
\hline Tenofovir Raltegravir Atazanavir Ritonavir & $1 \mathrm{NRTI}+1 \mathrm{INI}+1 \mathrm{PI}$ & 1 & 0.6 & 11,911 \\
\hline Lamivudine Lopinavir/Ritonavir & $1 \mathrm{NRTI}+1 \mathrm{PI}$ & 1 & 0.6 & 2,290 \\
\hline Lamivudine Darunavir Ritonavir & $1 \mathrm{NRTI}+1 \mathrm{PI}$ & 1 & 0.6 & 3,782 \\
\hline Lamivudine Atazanavir Ritonavir & $1 \mathrm{NRTI}+1 \mathrm{PI}$ & 11 & 6.3 & 6,209 \\
\hline Lamivudine Fosamprenavir Ritonavir & $1 \mathrm{NRTI}+1 \mathrm{PI}$ & 3 & 1.7 & 5,272 \\
\hline Lopinavir/Ritonavir & $1 \mathrm{PI}$ & 5 & 2.8 & 3,862 \\
\hline Darunavir Raltegravir Ritonavir & $1 \mathrm{PI}$ & 4 & 2.3 & 4,801 \\
\hline Fosamprenavir Ritonavir & $1 \mathrm{PI}$ & 3 & 1.7 & 4,054 \\
\hline Zidovudine/Lamivudine & $2 \mathrm{NRTI}$ & 2 & 1.1 & 211 \\
\hline Abacavir/Lamivudine Raltegravir & $2 \mathrm{NRTI}+1 \mathrm{INI}$ & 13 & 7.4 & 9,723 \\
\hline Abacavir/Lamivudine Nevirapine & $2 \mathrm{NRTI}+1 \mathrm{NNRTI}$ & 20 & 11.4 & 5,688 \\
\hline Abacavir/Lamivudine Efavirenz & $2 \mathrm{NRTI}+1 \mathrm{NNRTI}$ & 10 & 5.7 & 5,537 \\
\hline Zidovudine/Lamivudine Fosamprenavir Ritonavir & $2 \mathrm{NRTI}+1 \mathrm{PI}$ & 2 & 1.1 & 4,118 \\
\hline Abacavir/Lamivudine Lopinavir/Ritonavir & $2 \mathrm{NRTI}+1 \mathrm{PI}$ & 2 & 1.1 & 8,831 \\
\hline Abacavir/Lamivudine Darunavir Ritonavir & $2 \mathrm{NRTI}+1 \mathrm{Pl}$ & 5 & 2.8 & 8,869 \\
\hline Abacavir/Lamivudine Atazanavir & $2 \mathrm{NRTI}+1 \mathrm{PI}$ & 25 & 14.2 & 10,416 \\
\hline Abacavir/Lamivudine Atazanavir Ritonavir & $2 \mathrm{NRTI}+1 \mathrm{PI}$ & 8 & 4.5 & 8,225 \\
\hline Abacavir/Lamivudine Fosamprenavir & $2 \mathrm{NRTI}+1 \mathrm{PI}$ & 3 & 1.7 & 9,950 \\
\hline Abacavir/Lamivudine Fosamprenavir Ritonavir & $2 \mathrm{NRTI}+1 \mathrm{PI}$ & 5 & 2.8 & 8,447 \\
\hline Tenofovir/Emtricitabine Lopinavir/Ritonavir & $2 \mathrm{NRTI}+1 \mathrm{PI}$ & 5 & 2.8 & 9,558 \\
\hline Tenofovir/Emtricitabine Darunavir Ritonavir & $2 \mathrm{NRTI}+1 \mathrm{PI}$ & 3 & 1.7 & 8,826 \\
\hline Tenofovir/Emtricitabine Atazanavir Ritonavir & $2 \mathrm{NRTI}+1 \mathrm{Pl}$ & 10 & 5.7 & 8,968 \\
\hline Tenofovir/Emtricitabine Fosamprenavir Ritonavir & $2 \mathrm{NRTI}+1 \mathrm{PI}$ & 2 & 1.1 & 9,174 \\
\hline Zidovudine/Lamivudine Tenofovir & $3 \mathrm{NRTI}$ & 2 & 1.1 & 3,535 \\
\hline Abacavir/Lamivudine Rilpivirine & $3 \mathrm{NRTI}$ & 9 & 5.1 & 7,001 \\
\hline Abacavir/Lamivudine Tenofovir & $3 \mathrm{NRTI}$ & 1 & 0.6 & 7,864 \\
\hline Zidovudine/Lamivudine/Abacavir & $3 \mathrm{NRTI}$ & 9 & 5.1 & 6,019 \\
\hline Total & & 176 & 100 & 7,690 \\
\hline
\end{tabular}

Table III. Regimens characterized by a potential cardiovascular and/or metabolic toxicity [1] 


\begin{tabular}{|c|c|c|c|c|}
\hline \multirow{2}{*}{ Agents } & \multirow{2}{*}{ ART Regimens } & \multicolumn{3}{|c|}{ Patients } \\
\hline & & n. & $\%$ & Mean cost $(€)$ \\
\hline Dolutegravir Rilpivirine & 1NNRTI + INI & 68 & 9.8 & 8,712 \\
\hline Rilpivirine Darunavir Ritonavir & $1 \mathrm{NNRTI}+\mathrm{PI} / \mathrm{r}$ & 36 & 5.2 & 7,243 \\
\hline Lamivudine Dolutegravir & $1 \mathrm{NRTI}+\mathrm{INI}$ & 67 & 9.7 & 6,086 \\
\hline Lamivudine Atazanavir Ritonavir & $1 \mathrm{NRTI}+\mathrm{PI} / \mathrm{r}$ & 42 & 6.1 & 4,440 \\
\hline Tenofovir/emtricitabine & 2NRTI & 2 & 0.3 & 5,267 \\
\hline Tenofovir/emtricitabine Raltegravir & $2 \mathrm{NRTI}+\mathrm{INI}$ & 68 & 9.8 & 10,534 \\
\hline Tenofovir/emtricitabine Dolutegravir & $2 \mathrm{NRTI}+\mathrm{INI}$ & 55 & 7.9 & 11,209 \\
\hline Tenofovir/emtricitabine Rilpivirine & $2 N R T I+N N R T I$ & 63 & 9.1 & 8,036 \\
\hline Tenofovir/emtricitabine Nevirapine & $2 N R T I+N N R T I$ & 62 & 8.9 & 5,550 \\
\hline Tenofovir/emtricitabine Efavirenz & $2 \mathrm{NRTI}+\mathrm{NNRTI}$ & 10 & 1.4 & 5,699 \\
\hline Tenofovir/emtricitabine Nevirapine & $2 \mathrm{NRTI}+\mathrm{NNRTI}$ & 9 & 1.3 & 7,411 \\
\hline Tenofovir/emtricitabine Atazanavir & $2 \mathrm{NRTI}+\mathrm{PI}$ & 77 & 11.1 & 11,306 \\
\hline Tenofovir/emtricitabine Fosamprenavir & $2 N R T I+P I$ & 3 & 0.4 & 10,678 \\
\hline Tenofovir/emtricitabine Darunavir Ritonavir & $2 \mathrm{NRTI}+\mathrm{Pl} / \mathrm{r}$ & 15 & 2.2 & 9,740 \\
\hline Tenofovir/emtricitabine Atazanavir Ritonavir & $2 \mathrm{NRTI}+\mathrm{Pl} / \mathrm{r}$ & 31 & 4.5 & 9,563 \\
\hline Raltegravir Darunavir & $\mathrm{INI}+\mathrm{PI}$ & 13 & 1.9 & 11,903 \\
\hline Raltegravir Darunavir Ritonavir & $\mathrm{INI}+\mathrm{PI} / \mathrm{r}$ & 27 & 3.9 & 12,203 \\
\hline Lamivudine Darunavir Ritonavir & $\mathrm{NRTI}+\mathrm{Pl} / \mathrm{r}$ & 25 & 3.6 & 4,618 \\
\hline Darunavir Ritonavir & $\mathrm{Pl} / \mathrm{r}$ & 8 & 1.2 & 4,474 \\
\hline Atazanavir Ritonavir & $\mathrm{Pl} / \mathrm{r}$ & 12 & 1.7 & 4,296 \\
\hline Total & & 693 & 100.0 & 8,365 \\
\hline
\end{tabular}

Table IV. Alternative regimens in case of switch due to cardiovascular and/or metabolic toxicity [1]

\section{Current pharmaceutical expenditure}

In 2015, the actual pharmaceutical expenditure borne by the Center and generated by the 564 patients receiving antiretroviral drugs was $€ 4,042,983$, of which $€ 108,271$ for naïve patients and $€ 3,934,712$ for nonnaïve patients (Table $\mathrm{V})$. The mean pharmacological treatment cost was $€ 7,168 \pm €$ 2,605 (naïve patient: $€ 3,184 \pm 2,003$; nonnaïve patient $€ 7,424 \pm 2,426$ ). The pharmaceutical expenditure generated by the 111 patients receiving a triple therapy who could be treated with the nevirapine/raltegravir regimen was $€ 860,295$, with a mean cost of $€ 7,750 \pm 1,827$ (Table V). The 2 NRTIs + 1 PI combination resulted in the highest average cost $(€ 9,139 \pm 1,405)$, followed by 3 NRTIs $(€ 7,030, \pm 1,268)$ and 2 NRTIs +1 NNRTI $(€ 6,406, \pm 1,066)$. Cost differences between the 2 NRTIs +1 PI and the other two regimens were statistically significant ( $\mathrm{p}$ $<0.001$ ), while that between 3 NRTIs and 2 NRTIs + 1 NNRTI was not $(p=0.25)$. If costs at the individual patient level are analyzed, in 13 cases only $(11.7 \%)$ the use of a three-drug regimen resulted in an annual cost (range: $€$ $2,328-€ 5,408)$ lower than that of the nevirapine/raltegravir regimen $(€ 5,566)$.

However, the drug expenditure for the 176 patients for whom it would have been desirable to replace the current regimen at risk of toxicity, with one of those set out in the Italian Guidelines amounted to $€ 1,353,474$, with an average cost of $€ 7,690 \pm 2,634$ ) (Table V). Approximately $70 \%$ of these regimens consisted of 2 NRTIs +1 PI (n. $=70$; $€ 9,297 \pm 1,392), 2$ NRTIs +1 NNRTI (n. $=$ $30 ; € 5,638 \pm 864)$ and 3 NRTIs (n. $=21$; $€$ $6,291 \pm 1,151)$.

Finally, the other patients maintaining their current antiretroviral regimen without any change determined a cost of $€ 1,720,943$, with an average cost of $€ 7,082 \pm 2,473$ (Table V).

\section{Potential pharmaceutical expenditure}

Besides reporting the actual expenditure data for the antiretroviral drugs for the year 2015, Table $\mathrm{V}$ indicates the estimate for the same expenditure in light of the proposed optimization path (potential scenario). The expenditure associated with naive patients (patients who received the first treatment during 2015) remains exactly the same as that calculated for the actual scenario, since - by definition - naïve patients, not having been in virological suppression for at least 12 months, cannot fall within the selection criteria adopted by the optimization path. Therefore, the financial impact would occur only on non-naïve patients. The potential switch from a three- 


\begin{tabular}{|c|c|c|c|c|c|c|}
\hline \multirow{3}{*}{ Patients } & \multirow{3}{*}{ n. } & \multicolumn{4}{|c|}{ Treatment cost with cART $(€)$} & \multirow{3}{*}{ Delta } \\
\hline & & \multicolumn{2}{|c|}{ Actual scenario } & \multicolumn{2}{|c|}{ Potential scenario } & \\
\hline & & Total expenditure & Average cost & Total expenditure & Average cost & \\
\hline Naïve & 34 & 108,271 & 3,184 & 108,271 & 3,184 & 0 \\
\hline Non-naïve & 530 & $3,934,712$ & 7,424 & $3,806,584$ & 7,182 & $-128,128$ \\
\hline $\begin{array}{l}\text { - switch from a three-drug } \\
\text { to a two-drug regimen }\end{array}$ & 111 & 860,295 & 7,750 & 617,826 & 5,566 & $-242,469$ \\
\hline $\begin{array}{l}\text { - switch due to CV and/or } \\
\text { metabolic toxicity }\end{array}$ & 176 & $1,353,474$ & 7,690 & $1,467,815$ & 8,340 & 114,341 \\
\hline $\begin{array}{l}\text { - maintenance actual } \\
\text { regimen }\end{array}$ & 243 & $1,720,943$ & 7,082 & $1,720,943$ & 7,082 & 0 \\
\hline Total & 564 & $4,042,983$ & 7,168 & $3,914,855$ & 6,941 & $-128,128$ \\
\hline
\end{tabular}

Table V. Pharmaceutical expenditure charged to the Center: actual vs potential scenario

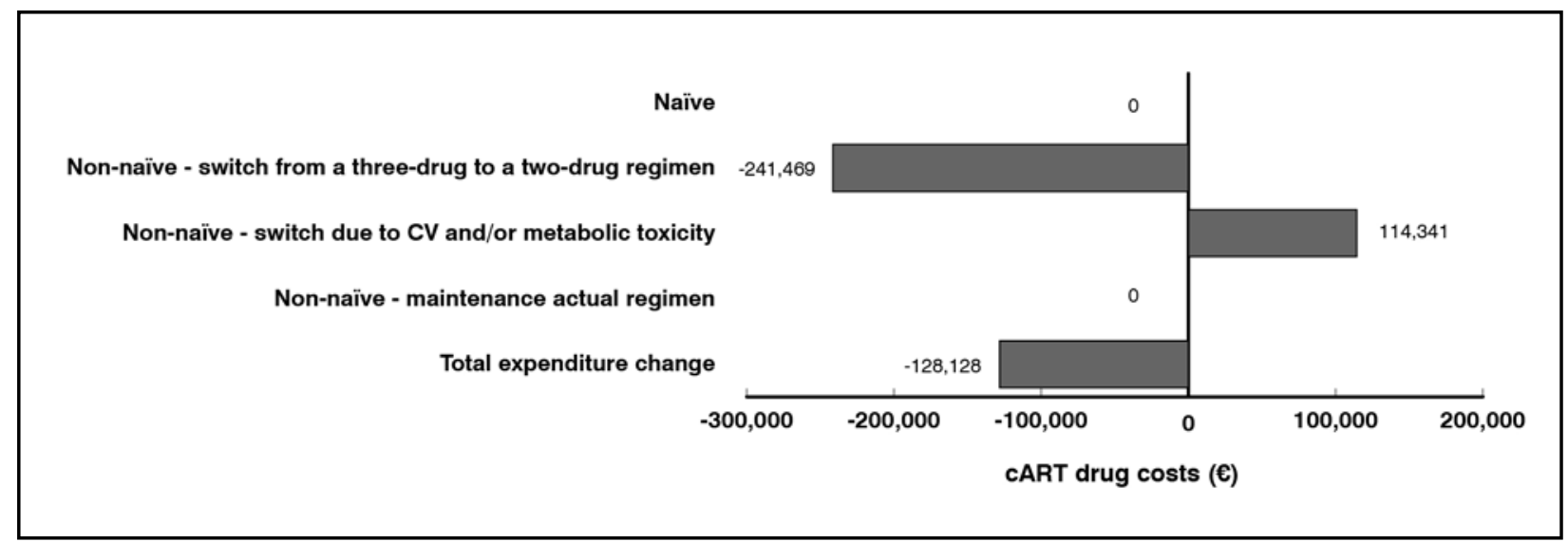

Figure 2. Financial impact of the optimization pathway (potential vs current scenario)

drug to a two-drug regimen (nevirapine/ raltegravir) would result, for the 111 patients treated, in a reduction in the cost borne by the Hospital Pharmacy of the Center of $€$ 242,469 . Conversely, the replacement of the regimens at risk of (cardiovascular and/or metabolic) toxicity with one of those indicated in the Italian Guidelines would cause, for the 176 patients treated, a potential increase in the cost borne by the Hospital Pharmacy of the Center of $€ 114,341$. The average annual cost associated with these alternative regimens was $€ 8,340 \pm 2,000$.

Overall, the adoption of the optimization pathway would result in a reduction in cART drug costs of $€ 128,128$ (-3.2\%) (Table $V$ and Figure 2).

\section{DISCUSSION}

The added value of this retrospective observational analysis - compared to the one previously conducted, again with reference to the Infectious Diseases Operating Unit of the Hospital "G.B. Rossi" in Verona [4] - consists in the fact that it assessed not only the financial impact of the switch from a three- drug to a two-drug regimen, but also the sustainability, on the part of the Center, of an antiretroviral therapy management pathway which, in the face of the potential cardiovascular and/or metabolic toxicities associated with current antiretroviral regimens, suggested instead, according to the indications of the Italian Guidelines [1], to preferably adopt regimens based on integrase inhibitors. In line with the previous analysis referred to the year 2014 [4], the first optimization pathway option - namely the switch from a threedrug to a two-drug regimen - would allow to reduce the Center-borne antiretroviral expenditure by around $€ 242,000$. This amount would allow the Center itself to cope with the increase in spending (around $€ 114,000$ ) associated with the second option of the optimization pathways - i.e. the replacement of potentially toxic regimens - as well as maintaining a "comfort zone" (about $€ 128,000$ ) with which to cope with any therapy switches that might become necessary.

The clinical rationale that makes it possible to switch from a three-drug regimen to the nevirapine/raltegravir combination relies on the results of two recent clinical trials $[5,6]$. 
Both were conducted with the aim of evaluating the efficacy of a dual therapy consisting of 1 NNRTI +1 INI in place of HAART regimens as maintenance treatment of HIVinfected patients in virological suppression. During the period September 2009-January 2012 , all patients referred to the centers of Besançon and Nantes with HIV infection, with a viral load $<50$ copies $/ \mathrm{ml}$ for more than six months and without prior exposure to INIs were switched from a three-drug regimen to the combination nevirapine (400 mg/ day) and raltegravir (400 $\mathrm{mg}$ BID) [5]. The 36 enrolled patients, followed for a maximum of three years, maintained a viral load $<50$ copies $/ \mathrm{ml}$, without the occurrence of grade 3 or 4 adverse events [5]. The subsequent Italian study replicated and validated de facto the previous French analysis [6]. After a 32-month median time, $89.6 \%$ of the 77 patients enrolled in the nevirapine/raltegravir regimen were in virological suppression, while $6.5 \%$ experienced a virologic failure and $3.9 \%$ discontinued the treatment due to adverse events (skin rash or hepatic toxicity) [6]. The results of these experiences show that, in a well-defined case record of patients, the switch to a nevirapine/raltegravir therapy allows the long-term maintenance of an adequate virological suppression.

As indicated in the methods, the identification of the toxic regimens was performed by examining at the individual patient level the regimen administered during the observation year. Table III details the regimens characterized by a risk of cardiovascular and/or metabolic toxicity for the 176 subjects for whom it would have been possible to switch the therapy with a regimen characterized by a reduced risk of toxicity. $71.6 \%$ of these regimens (126 out of 176) contain abacavir or lopinavir, the exposure to which is a predictor of cardiovascular risk [7]. The alternative regimens, listed in Table IV and identified according to the indications of the Italian Guidelines, have always been identified at the individual patient level. In $49.4 \%$ of cases ( 87 out of 176 ), only one alternative method was identified; in the remaining cases, the choice was assumed between multiple treatments with different treatment costs. In $28.4 \%$ of cases, the choice could fall on more than seven alternatives. For this reason, the economic impact associated with the switch to a low cardiovascular and/or metabolic toxicity regimen may be subject to variations. Had we considered, instead of the average cost, the minimum cost of the alternatives indicated for each patient $(€ 6,888)$, the "comfort zone" would have increased to $€ 383,652$ compared to the base case; whereas, had we considered the maximum cost of the alternatives indicated for each patient $(€ 9,688)$, the optimization pathway would have resulted in an increase in the cost of the antiretroviral drugs borne by the Center of $€ 109,201$. However, in the absence of the financial effect resulting from the switch from a three-drug to a two-drug regimen $(-€ 242,469)$, this increase in expenditure for the Center would have been higher (€ 351,671).

Local cost containment HIV strategies has been investigated considering different approaches (i.e., generic drugs, switch from a three-drug to a two-drug regimen or monotherapies) and costs (cART, hospitalization, outpatient activities, adverse events' management). For this reason it is difficult to compare the present results with those of already published studies. The analysis, conducted by Angeletti et al. [8], shows how the most cost containing strategy would be the use of generic drugs, followed by simplification to monotherapy. A second analysis investigated the budget impact of ART simplification to less drug regimens over a 3-year horizon (costs referred to 2013) [9]. The Authors considered 4 simplification scenarios: i) deintensifying only PI-based triple therapies over 1 year period ii) de-intensifying only PIbased triple therapies over a 3-year period, iii) de-intensifying PI-based triple therapies and NRTIs + efavirenz over 1 year period and iv) de-intensifying PI-based triple therapies and NRTIs + efavirenz over a 3-year period. Over a 3-year period, ART cost decreased between $€ 23.1$ million and $€ 44.3$ million considering different scenarios. A third analysis was conducted to evaluating the impact of treatment simplification of atazanavir (ATV) + ritonavir ( $\mathrm{r}$ ) + lamivudine (3TC) in virologically suppressed patients receiving ATV $+r+2$ nucleoside reverse transcriptase inhibitors (NRTIs) [10]. The perspective of the Italian National Health Service (NHS) was considered. The antiretroviral treatment simplification strategy considered would lead to lower costs for the Italian NHS in a 5-year time horizon between $€-28.7$ million and $€$ -16.0 million.

Although the comparison sought to provide a realistic scenario of the economic impact following the adoption of a specific optimization pathway for the cART regimens administered for the treatment of HIV patients with the aim of maintaining the therapeutic effectiveness also over the long term, the results presented here must be interpreted in light of some observations.

First of all, the hypothesis of being able to administer the alternative regimens suggested by the optimization path to the same pa- 
tients treated in 2015 with cART drugs. This assumption is the basis of this analysis, since it was not possible to create a real control group treated with the alternative regimens hypothesized by the optimization pathway. In light of this necessary compromise, in addition to the effectiveness data reported in the literature $[5,6]$ and by the Italian Guidelines [1], further criteria supporting the switch to an alternative antiretroviral regimen were sought. For example, in the switch towards a two-drug regimen, since this would reduce the risk of long-term toxicity [11-15], it was established that patients - besides being in virological suppression - had also to report a toxicity associated with the HAART therapy. A second critical aspect may be represented by the average cost considered in order to enhance the nevirapine/raltegravir regimen, or the alternative ones (cardiovascular and/ or metabolic toxicity). Such average cost, in fact, does not derive from real-world consumption data, as was the case with the regimens actually administered by the Center, but is based on the purchase cost of the molecules, borne by the Center's pharmacy, and the relevant dosages indicated in the literature, assuming that the duration of treatment is one year. In actual fact, however, this choice may have overestimated or underestimated the results found here, since - by adopting such administration regimens - a total compliance with the administered treatment was assumed, thus excluding the presence of cases of over- or under-consumption, contemplated instead for the actual spending. The present analysis collected information only on the cART costs; other cost items were not considered (eg. hospitalizations, specialist visits, etc.), which however are potentially interesting for those wishing to examine in detail the knowledge of the resources required by HIV.

At last, a final limitation could be the representativeness of these results on a national scale, since they are referred to a single center located in Northern Italy. The verification of a possible variability of the data found here could be carried out only in the presence of similar analyses, conducted in other Italian geographic areas.

\section{CONCLUSIONS}

This observational analysis was carried out with the objective of estimating the financial impact of an optimization pathway of the antiretroviral therapy, aimed at maintaining its effectiveness over time. In light of the results presented, albeit with some limitations, it would seem possible to state that the optimization pathway proposed here is a valid therapeutic option in the maintenance treatment of suppressed HIV-1 patients, since it can make sustainable the costs of the cART therapy currently charged to the Italian NHS, while ensuring the maintenance over time of adequate levels of effectiveness and safety.

\section{Funding}

No financial support was received for this submission.

\section{Conflict of interest}

The authors have no conflict of interest concerning the topics of this article.

\section{REFERENCES}

1. Ministero della Salute - SIMIT. Linee Guida Italiane sull'utilizzo dei farmaci antiretrovirali e sulla gestione diagnostico-clinica delle persone con infezione da HIV-1. November 2016. Available at http://www.salute.gov.it/ imgs/C_17_pubblicazioni_2545_allegato.pdf (last accessed April 2017)

2. Negredo E, Bonjoch A, Clotet B. Benefits and concerns of simplification strategies in HIV-infected patients. $J$ Antimicrob Chemother 2006; 58: 235-42; https://doi.org/10.1093/jac/dkl191

3. Margolis AM, Heverling H, Pham PA, et al. A Review of the Toxicity of HIV Medications. J Med Toxicol 2014; 10: 26-39; https://doi.org/10.1007/s13181-013-0325-8

4. Ravasio R, Rigo F, Lattuada E, et al. Economic evaluation of nevirapine plus raltegravir as maintenance antiretroviral therapy in virologically suppressed HIV-1 infected patients. GRHTA 2016; 3: 110-4

5. Reliquet V, Chirouze C, Allavena C, et al. Nevirapine-raltegravir combination, an NRTI and PI/r sparing regimen, as maintenance antiretroviral therapy in virologically suppressed HIV-1-infected patients. Antivir Ther 2014; 19: 117-23; https://doi.org/10.3851/IMP2691

6. Calcagno C, Montrucchio A, Capetti G, et al. Raltegravir Plus Nevirapine as Maintenance Antiretroviral Therapy inHIV-Positive Patients: Safety, Efficacy and Pharmacokinetics. Curr HIV Res 2016; 14: 54-60 
7. Friis-Møller N, Ryom L, Smith C, et al.; D:A:D study group. An updated prediction model of the global risk of cardiovascular disease in HIV-positive persons: The Data-collection on Adverse Effects of Anti-HIV Drugs (D:A:D) study. Eur J Prev Cardiol 2016; 23: 214-23; https://doi.org/10.1177/2047487315579291

8. Angeletti C, Pezzotti P, Antinori A, et al. Antiretroviral treatment-based cost saving interventions may offset expenses for new patients and earlier treatment start. HIV Med 2014; 15: 165-74; https://doi.org/10.1111/hiv.12097

9. Restelli U, Andreoni M, Antinori A, et al. Budget impact analysis of antiretroviral less drug regimen simplification in HIV-positive patients on the Italian National Health Service. Clinicoecon Outcomes Res 2014; 6: 409-14; https:// doi.org/10.2147/CEOR.S68101

10. Restelli U, Fabbiani M, Di Giambenedetto S, et al. Budget impact analysis of the simplification to atazanavir + ritonavir + lamivudine dual therapy of HIV-positive patients receiving atazanavir-based triple therapies in Italy starting from data of the Atlas-M trial. Clinicoecon Outcomes Res 2017; 9: 173-9; https://doi.org/10.2147/CEOR.S127097

11. Reynes J, Trinh R, Pulido F, et al. Lopinavir/ritonavir combined with raltegravir or tenofovir/emtricitabine in antiretroviral-naïve subjects: 96-week results of the PROGRESS study. AIDS Res Hum Retroviruses 2013; 29: 256-65

12. Nozza S, Galli L, Antinori A, et al. Maraviroc $150 \mathrm{mg}$ QD plus lopinavir/ritonavir, a NRTIs-sparing regimen for naïve patients: preliminary 48-weeks results. In Abstracts of the 6th International AIDS Society, Rome, Italy, 2011, July 17-20. Abstract CDB325.

13. Calcagno A, Nozza S, Gonzalez de Requena D, et al. Pharmacokinetics of maraviroc administered at $150 \mathrm{mg}$ once daily in association with lopinavir/ritonavir in HIV-positive treatmentnaïve patients. J Antimicrob Chemother 2013; 68: 1686-8; https://doi.org/10.1093/jac/dkt074

14. Boyd MA, Kumarasamy N, Moore CL, et al. Ritonavir-boosted lopinavir plus nucleoside or nucleotide reverse transcriptase inhibitors versus ritonavir-boosted lopinavir plus raltegravir for treatment of HIV-1 infection in adults with virological failure of a standard first-line ART regimen (SECOND-LINE): a randomised, open-label, noninferiority study. Lancet 2013; 381: 2091-9; https://doi.org/10.1016/S0140-6736(13)61164-2

15. Reliquet V, Allavena C, Morineau-Le Houssine P, et al. Twelve-year experience of nevirapina use: benefits and convenience for long-term management in a French cohort of HIV-1-infected patients. HIV Clin Trials 2010; 11: 110-7; https://doi.org/10.1310/hct1102-110 